\title{
Congenital myasthenic syndromes: recent advances
}

\author{
David Beeson
}

\begin{abstract}
Purpose of review
Congenital myasthenic syndromes (CMS) are a group of heterogeneous inherited disorders caused by mutations in genes encoding proteins essential for the integrity of neuromuscular transmission. This review updates the reader on recent findings that have expanded the phenotypic spectrum and suggested improved treatment strategies.
\end{abstract}

\section{Recent findings}

The use of next-generation sequencing is continuing to unearth new genes in which mutations can give rise to defective neuromuscular transmission. The defective transmission may be part of an overall more complex phenotype in which there may be muscle, central nervous system or other involvement. Notably, mutations in series of genes encoding presynaptic proteins are being identified. Further work on mutations found in the AGRN-MUSK acetylcholine receptor clustering pathway has helped characterize the role of LRP4 and broadened the phenotypic spectrum for AGRN mutations. Mutations in another extracellular matrix protein, collagen $13 \mathrm{Al}$ and in GMPPB have also been found to cause a CMS. Finally, there are an increasing number of reports for the beneficial effects of treatment with $\beta 2$-adrenergic receptor agonists.

\section{Summary}

Recent studies of the CMS illustrate the increasing complexity of the genetics, pathophysiological mechanisms and the need to tailor therapy for the genetic disorders of the neuromuscular junction.

\section{Keywords}

collagen 13A1, congenital myasthenic syndromes, N-glycosylation pathway, salbutamol, presynaptic

\section{INTRODUCTION}

Congenital myasthenic syndromes (CMS) are a group of heterogeneous inherited disorders caused by mutations in genes encoding proteins that are essential for maintaining the integrity of neuromuscular transmission [1,2]. The syndromes share the clinical feature of fatigable weakness, but age at onset, presenting symptoms, distribution of weakness and response to treatment vary depending on the molecular mechanism resulting from the genetic defect. To date, at least 24 different genes are known to cause CMS (Table 1 ; [3]). Next-generation sequencing (NGS) is helping to spread the net in identifying causative genes that now include proteins that are ubiquitously expressed but their defective function is most apparent at the neuromuscular junction, and presynaptic proteins in which multiple functional deficits are present, of which defective neuromuscular transmission is one.

In this review, we report the most recent findings in CMS regarding novel causative genes, notable observations for the better established CMS subtypes and an update on treatment. Readers are referred to other reviews for a more general overview of CMS $[1,4]$.

\section{PRESYNAPTIC SYNDROMES}

Until recently, mutations in CHAT, the gene encoding choline acetyltransferase, were the only presynaptic cause of a CMS. The hallmark phenotypic feature for patients with CHAT mutations is episodic apnea that can be fatal. New reports support the original findings of Ohno et al. [5] that characterized the mutations as affecting the catalytic activity of the enzyme, the expression levels of the enzyme or a combination of the two [6]. This leads to a use-dependent reduction in the filling of acetylcholine (ACh) molecules into the synaptic vesicles of the motor nerve terminal and thus a reduction in the quantal release of ACh into the synaptic cleft.

Neurosciences Group, Nuffield Department of Clinical Neurosciences, Weatherall Institute of Molecular Medicine, University of Oxford, The John Radcliffe Hospital, Oxford, UK

Correspondence to Professor David Beeson, Neurosciences Group, Nuffield Department of Clinical Neurosciences, Weatherall Institute of Molecular Medicine, John Radcliffe Hospital, Oxford OX3 9DS, UK.

Tel: +44 1865 222311; fax: +44 1865222402 ;

e-mail: david.beeson@ndcn.ox.ac.uk

Curr Opin Neurol 2016, 29:565-571

DOI:10.1097/WCO.0000000000000370 


\section{KEY POINTS}

- Next-generation sequencing continues to help uncover new CMS disease genes and broaden the phenotypic spectrum for these syndromes.

- Myasthenic weakness may be only one component of a broader disease phenotype but its recognition is important as it will be amenable to treatment with drugs that enhance neuromuscular transmission.

- The use of refined treatment combinations may maximize the benefit to patients with congenital myasthenia.

What appears puzzling is why the effects of the CHAT mutations are restricted to the neuromuscular junction and the reduction of ACh release is not apparent in central cholinergic synapses. The use of NGS is now uncovering mutations in many more presynaptic proteins, but in these cases a varying degree of central problems are evident. Release of the neurotransmitter ACh into the synaptic cleft is dependent on a multistep cycle in which ACh is synthesized from acetyl CoA and choline, is transported into vesicles that congregate adjacent to the nerve terminal, is exocytosed into the synaptic cleft through fusion of the vesicles with the motor terminal membrane that involves the SNARE protein complex and then in the synaptic cleft ACh is rapidly broken down and the reuptake of choline into the nerve terminal occurs largely through the sodium-dependent high-affinity choline transporter (Fig. 1). There is the potential for diminished or altered function in many of these steps to affect levels of neurotransmitter available to bind the acetylcholine receptor (AChR) and thus to cause myasthenic symptoms. The use of NGS is now revealing that many genes encoding proteins in this pathway can harbor mutations that lead to myasthenic features in the patient. Contrasting with the majority of CMS that are recessive disorders, dominant mutations have been identified for SNARE complex protein SNAP25B [7"'] and for synaptotagmin (SYT2) [8"',9] that locates to synaptic vesicles and acts as a calcium sensor for vesicular fusion and neurotransmitter release [10]. The de novo mutation in SNAP25B led not only to reduced quantal release from the motor nerve terminal but was also associated with severe developmental delay, ataxic gait and ataxic dysarthria. Treatment with 3,4-diaminopyridine (3,4-DAP) improved patient strength. Mutations in SYT2, identified in two families (p.Asp307Ala and p.Pro308Leu, respectively), had features of a motor neuropathy and a presynaptic disorder resembling
Table 1. Congenital myasthenic syndromes subtypes and associated genes

\begin{tabular}{|c|c|}
\hline $\begin{array}{l}\text { Congenital myasthenic } \\
\text { syndrome }\end{array}$ & Gene \\
\hline \multicolumn{2}{|l|}{ Ubiquitously expressed proteins } \\
\hline \multicolumn{2}{|l|}{ N-glycosylation pathway } \\
\hline GFPT1 & GFPT1 \\
\hline DPAGT1 & DPAGT1 \\
\hline${ }^{a} A L G 2$ & ALG2 \\
\hline${ }^{a} A L G 14$ & ALG14 \\
\hline GMPPB & GMPPB \\
\hline \multicolumn{2}{|l|}{ Propyl-oligopeptidase } \\
\hline aPREPL & PREPL \\
\hline \multicolumn{2}{|c|}{ Proteins with defined function at the neuromuscular junction } \\
\hline \multicolumn{2}{|c|}{ Presynaptic } \\
\hline Choline acetyltransferase & CHAT \\
\hline${ }^{a}$ Synaptotagmin & SYT2 \\
\hline${ }^{a} \mathrm{SNAP} 25 \mathrm{~B}$ & SNAP25B \\
\hline \multicolumn{2}{|l|}{ Synaptic } \\
\hline Endplate AChE deficiency & COLQ \\
\hline aLaminin $\beta 2$ deficiency & $\angle A M B 2$ \\
\hline Agrin & AGRN \\
\hline${ }^{a}$ Collagen $13 \mathrm{~A} 1$ & COLI3Al \\
\hline \multicolumn{2}{|l|}{ Postsynaptic } \\
\hline $\begin{array}{l}\text { Primary acetylcholine } \\
\text { receptor deficiency }\end{array}$ & $\begin{array}{c}\text { CHRNA 1, CHRNB 1, } \\
\text { CHRND, CHRNE }\end{array}$ \\
\hline Escobar syndrome & CHRNG \\
\hline Rapsyn & RAPSN \\
\hline DOK7 & DOK7 \\
\hline Slow-channel syndrome & $\begin{array}{c}\text { CHRNA 1, CHRNB 1, } \\
\text { CHRND, CHRNE }\end{array}$ \\
\hline Fast-channel syndrome & $\begin{array}{c}\text { CHRNA 1, CHRNB 1, } \\
\text { CHRND, CHRNE }\end{array}$ \\
\hline aLow-conductance syndrome & CHRNE \\
\hline${ }^{a}$ MuSK & MUSK \\
\hline aplectin deficiency & PLEC 1 \\
\hline aNa-channel myasthenia & SCN4A \\
\hline aLRP4 & LRP4 \\
\hline
\end{tabular}

Source: Updated from Rodrigues-Cruz, Palace, Beeson Curr Opin Neurol 2014. AChE, acetylcholinesterase.

${ }^{a}$ Accounts for rare CMS syndromes. Adapted from [3].

the Lambert-Eaton myasthenic syndrome. Characteristically, neurophysiology showed low amplitude of compound muscle action potential (CMAP) with postexercise amplitude facilitation. Clinical features included childhood onset foot deformities, lower limb muscle atrophy and weakness, and absent deep tendon reflexes. Other candidate proteins in which mutations might result in presynaptic disorders would include members of the SNARE complex such as synaptobrevin and syntaxin, the high-affinity 


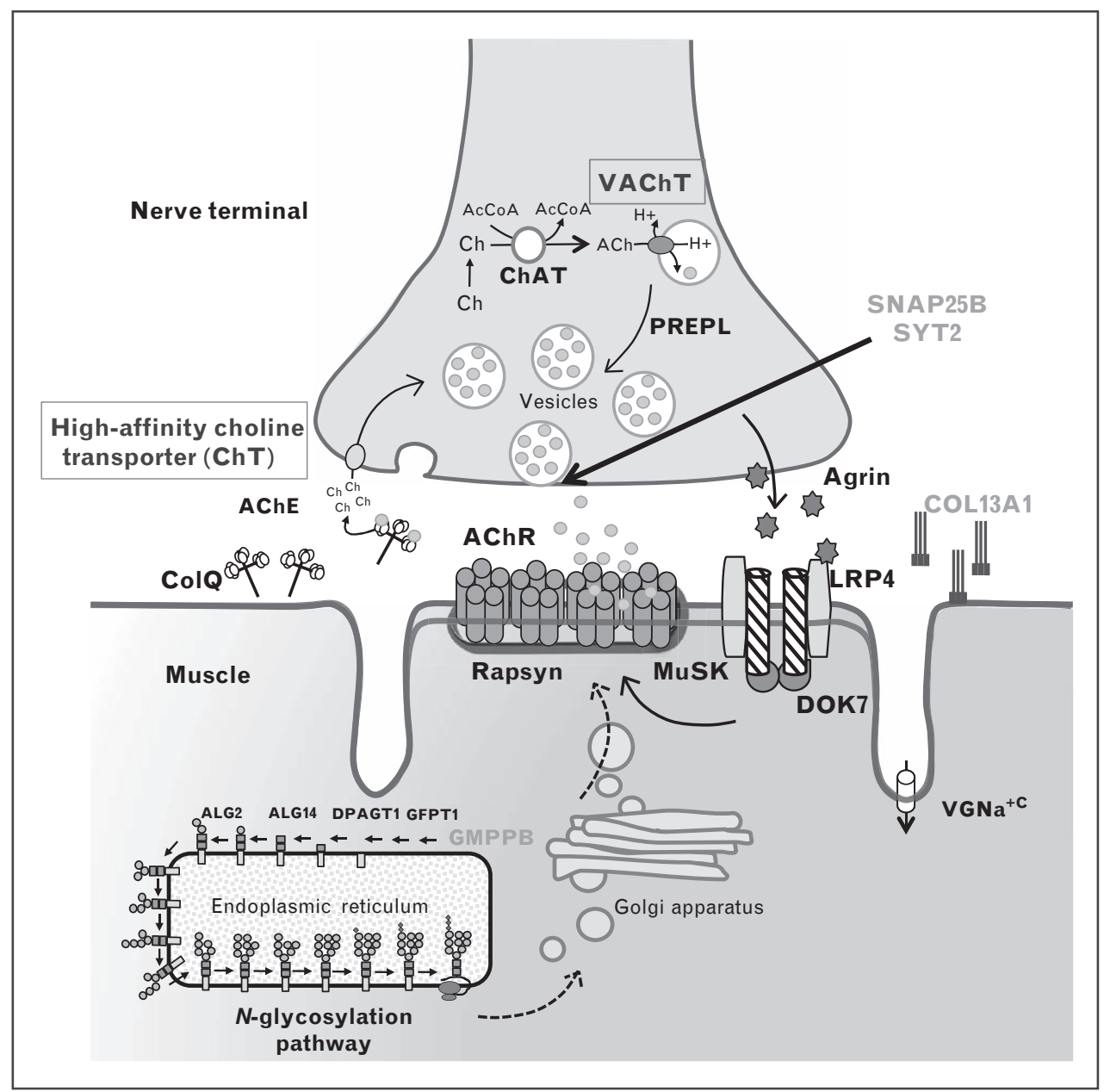

FIGURE 1. The neuromuscular junction and the principle molecules involved in congenital myasthenic syndromes. The spectrum of molecular mechanisms involved in congenital myasthenic syndrome. Common mutated proteins, newly identified disease genes and presynaptic candidate genes are illustrated. Newly identified disease genes are labeled in grey (SNAP25B, SYT2, COL13A1 and GMPPB). Candidate presynaptic genes are boxed and labeled in black. The high-affinity choline transporter is encoded by SLC5A7, and the vesicular acetylcholine transporter is encoded by SLC18A3. GMPPB, DPAGT1, ALG2, ALG14 and GFPT1 are represented at the postsynaptic endoplasmic reticulum, in a simplified view of the $N$ glycosylation pathway, together with the posttranslational modifications of the saccharide structure of the acetylcholine receptor and other neuromuscular junction proteins inside the endoplasmic reticulum and Golgi apparatus, before reaching the muscle cell surface as mature proteins. AChE, acetylcholinesterase. Source: Modified from Rodrigues-Cruz, Palace, Beeson Curr Opin Neurol 2014 [3].

choline transporter, and the vesicular ACh transporter. It is not clear yet what phenotype dysfunction in these proteins would lead to, whether defective neuromuscular transmission would be the predominant symptom, or how they would respond to current therapies.

\section{BASAL LAMINA-ASSOCIATED SYNDROMES}

Mutations in the collagen-like tail subunit, which anchors the asymmetric form of acetylcholinesterase at the neuromuscular junction, are established as a major cause of CMS [11]. Other basal lamina-associated CMS are rare. A single case of deficiency of laminin $\beta 2$ (LAMB2) due to heteroallelic frameshift mutations has been reported [12]. A recent report has identified an additional basal lamina-associated gene in which mutations can give rise to a myasthenic syndrome [13"']. COL13A1 is a transmembrane protein concentrated at the neuromuscular junction with an ectodomain that can be cleaved and shed into the synaptic cleft [14]. Studies in mice lacking col13a1 reveal abnormal synaptic maturation and defective presynaptic and postsynaptic functions [15]. Mutations in COL13A1 have now been identified in two independent families [13"']. In 
one, there was a homozygous frameshift c.1171delG, p.Leu392S $f s^{*} 71$, and in the other a homozygous splice site mutation c.523-1delG that is predicted to also cause a frameshift p.Gly175V $f s^{*} 20$. Onset of the disorder was at birth with respiratory and feeding difficulties, slight dysmorphic facial features and marked ptosis but normal eye movements. Analysis of a patient muscle biopsy showed the expected complete lack of COL13A1 at endplates, but normal staining for other endplate proteins such as DOK7, MUSK, RAPSN and the AChR. Functional studies were in keeping with results from col13a1 $1^{-/-}$mice that suggest a defect in synaptic maturation and in keeping with these observations that patients were found not to respond to pyridostigmine but to benefit from the $\beta 2$-adrenergic receptor agonist salbutamol. The findings emphasize the crucial role of extracellular matrix proteins, other than those in the agrin (AGRN) pathway, for the formation and maintenance of the synapse, and highlight the importance of collagen XIII in neuromuscular junction cytoarchitecture and neurotransmission.

\section{The agrin pathway}

NGS is also helping to identify mutations in genes encoding some of the larger and more complex proteins at the neuromuscular junction that might previously have gone undetected. AGRN is a large extracellular matrix proteoglycan that has several isoforms of which the neural isoform plays the key role in maintaining synaptic structure through binding LDL receptor related protein 4 (LRP4) and activating MUSK $[16,17]$. New cases of CMS due to AGRN mutations have been identified with distal muscle weakness and atrophy resembling a distal myopathy [18"]. Patients had decrement of CMAP on repetitive nerve stimulation but also showed postexercise increment of CMAP, though this varied depending upon which muscle was sampled, suggesting an additional presynaptic effect due to the AGRN mutations. No clear genotype-phenotype correlation was apparent, although the identification of mutations in the N-terminal AGRN domain emphasizes the importance of screening the whole gene for pathogenic variants.

LRP4 is the most recent of the key molecules for the development and maintenance of the neuromuscular junction to be identified. AGRN binds to LRP4 forming a ternary complex with MUSK that triggers phosphorylation of the MUSK intracellular kinase domain. A second family with two affected sisters has now been identified with a homozygous mutation in LRP4 (c.3698A > C, p.Glu1233Ala) [19"].
As might be predicted, the mutation is found to inhibit AGRN-induced upregulation of MUSK signaling, thus disrupting maintenance of the neuromuscular junction. The location of this mutation at the edge of the third $\beta$-propeller domain of LRP4 and the mutations p.Glu1233Lys and p.Arg1277His [20] suggest that this is a restricted region for CMS-causing mutations within LRP4, whereas mutations in other regions of the gene may give rise to Cenani-Lenz syndactyly syndrome [21] or sclerosteosis-2 [22].

\section{CONGENITAL MYASTHENIC SYNDROME DUE TO DEFECTS IN THE GLYCOSYLATION PATHWAY}

The asparagine-linked (N-linked) glycosylation pathway is a ubiquitous process in eukaryote cells defined by the sequential attachment of sugar moieties that are then linked to an amino acid residue in a protein. Membrane-bound and exported proteins are modified in the endoplasmic reticulum by the addition and processing of glycans that can be crucial for protein folding, assembly, stability, intracellular transport and function. Mutations in components within this pathway produce a spectrum of severe multisystemic disorders known as congenital disorders of glycosylation [23-25]. NGS has helped uncover mutations in genes that contribute to the early steps of the N-linked glycosylation pathway as a cause of CMS [26-28]. Mutations in these genes can result in severe multisystem disorders, so the reasons why mutations in ALG2, ALG14, DPAGT1 and GFPT1 that are ubiquitously expressed result in symptoms that are largely restricted to the neuromuscular junction dysfunction is unclear. Indeed, mass spectrometric studies on the $\mathrm{N}$-glycomes of muscle cells derived from GFPT1 CMS patients showed no global difference between the patient cells and control muscle cells [29]. The neuromuscular junction is known to be heavily glycosylated, and it may be that this dynamic synapse and the AChR itself are especially sensitive to only modest flaws in the pathway [30]. When mutations in GFPT1 were first identified as a cause of CMS, a number of patients were found to have the transversion c. ${ }^{*} 22 \mathrm{C}>\mathrm{A}$ in the $3^{\prime}$-untranslated region of the gene. It was not clear how this change in the untranslated region of the gene was pathogenic, but a recent report identified that it creates a microRNA binding site and that binding of microRNA206* reduced GFPT1 expression [31"“]. Thus, the report highlights that variants that create or disrupt microRNA binding sites in untranslated regions can be pathogenic and should be considered in genetic screens. 


\section{Congenital myasthenic syndrome due to mutations on GMPPB}

$G M P P B$ encodes the enzyme mannose-1-phosphate guanyltransferase beta (GMPPB) that catalyses the formation of GDP-mannose from mannose1-phosphate and GTP [32]. GDP-mannose is used as building block in multiple glycosylation steps including N-linked and O-linked glycosylation. Previously, mutations in GMPPB have been associated with muscular dystrophy dystroglycanopathies in which defective $O$-glycosylation of $\alpha$-dystroglycan leads to dystrophic features often accompanied by a variable degree of structural brain and eye abnormalities [33,34]. A recent report now expands the phenotype generated by mutations in GMPPB to include a CMS [35"'].

Some patients with GMPPB mutations have no detectable neuromuscular junction abnormalities, whereas in others fatigable muscle weakness with a characteristic neuromuscular junction transmission defect is the main symptom. However, all patients tested have reduced glycosylation of $\alpha$ dystroglycan. Thus, in this CMS subtype, there is a bridge between a myasthenic syndrome and a dystroglycanopathy [35"']. Patients with GMPPBCMS have a similar phenotype to other CMS subtypes harboring mutations within the early stages of the glycosylation pathway, with a characteristic limb girdle pattern of muscle weakness, onset in childhood or later and often absence of ptosis and sparing of eye and facial weakness. Notably, electromyography (EMG) abnormalities may be absent in eye and facial muscles but present in the muscle groups that are weak. Additional features shared with the dystroglycanopathies include myopathic features, raised creatine kinase (CK) levels and mild cognitive delay. A raised CK reading is unusual for CMS, though mildly raised levels may be seen in GFPT1 CMS and occasionally in the slow-channel syndrome, and therefore the combination of markedly raised $\mathrm{CK}$ and decrement on EMG would be suggestive of GMPPB-CMS [36]. Recognition of CMS in patients with dystroglycanopathies is important in that it provides the opportunity for effective symptomatic treatment for the neuromuscular junction component of the disorder.

\section{MUTATIONS IN ESTABLISHED FORMS OF CONGENITAL MYASTHENIC SYNDROME}

Variation in phenotypic features and mutations of note continue to be identified in established forms of CMS. Two cases of recessive mutations in SCN4A, the voltage-gated sodium channel that propagates the membrane depolarization at the neuromuscular junction into an action potential, have been reported $\left[37^{*}, 38^{*}\right]$. The mutations p.Arg1454Trp and p.Arg1457His are in the DIVS4 region of the protein and cause delayed recovery from inactivation states, which could potentially translate into myasthenic weakness through use-dependent inactivation. The p.Arg1454Trp mutation generates a phenotype in which CMS and periodic paralysis overlap [38"].

NGS is increasingly picking up variants in known CMS-associated genes. For example, a screen of a large cohort of patients with fetal akinesia identified heteroallelic mutations in CHRND (p.8Cys257Arg and p.Val439Trpfs*11) leading to a severe CMS [39]. Further, a screen of late-presenting myasthenia cases suggestive of seronegative myasthenia gravis led to the identification of seven CMS cases out of a cohort of 25 with mutations in RAPSN or CHRNA1, several of whom had previously been treated for autoimmune myasthenia [40]. As the clinical use of NGS expands, the emphasis of interpreting the data generated will move from the detection of sequence variants to determining the pathogenicity of variants and sufficient understanding of the molecular mechanisms to formulate an appropriate therapeutic strategy.

\section{TREATMENT}

Current therapies for CMS include cholinesterase inhibitors such as pyridostigmine, the potassium channel blocker - 3,4-DAP, AChR open channel blockers - fluoxetine or quinidine and the $\beta 2$-adrenergic receptor agonists - ephedrine and salbutamol. Response to treatment depends on the CMS subtype. However, the use of salbutamol or ephedrine alone or combined with other drugs is increasingly being reported as beneficial for many CMS subtypes. These drugs can have a dramatic beneficial effect for patients with DOK7 mutations, although, unlike the near immediate effects of pyridostigmine or 3,4-DAP, the benefit builds up over weeks or months [41-44]. Based on the work with DOK7 CMS, it is hypothesized that the $\beta 2$-agonists should be beneficial for all mutations that impair the AGRN-LRP4-MUSK-DOK7 signaling pathway [41]. It is reassuring therefore that patients with mutations in LRP4 [19"], MUSK [45] and AGRN [18"] are reported to benefit from salbutamol, although the response for AGRN mutations may be less marked. A more recent study reports a marked improvement following the addition of $\beta 2$-agonists therapy to patients with severe weakness from ACh deficiency due to CHRNE mutations on optimum treatment with pyridostigmine and 3,4-DAP [46"']. Studies on mouse models in which components of the AGRN pathway were 'knocked 


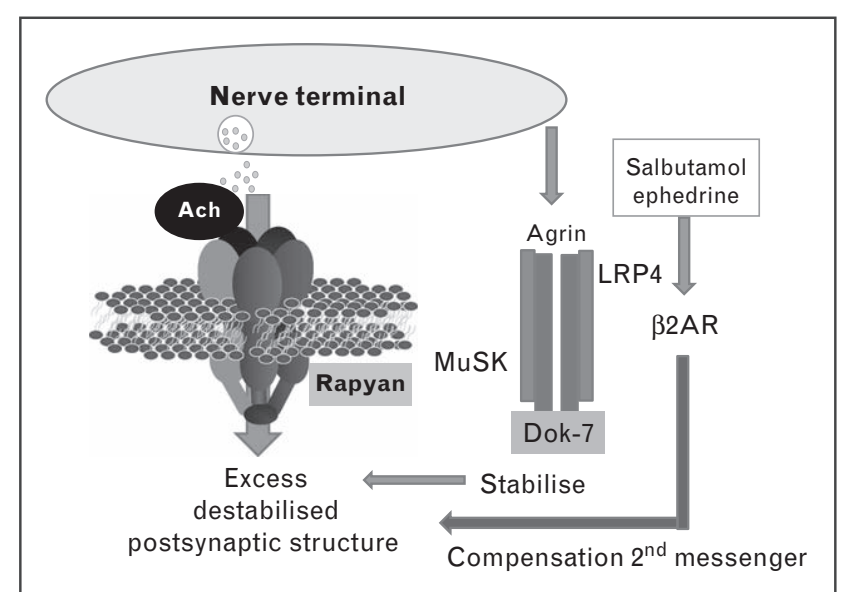

FIGURE 2. Hypothetical model of the action of $\beta 2$-agonists at the neuromuscular junction. Diagrammatic model of disruption of endplate structure caused by excess cholinergic transmission counteracted by AGRN-LRP4-MUSK-DOK7 signaling that stabilizes endplate structures. It is proposed that $\beta 2$-agonist can partially compensate when signaling in this pathway is impaired. Similarly, it is proposed that $\beta 2$-agonists can help stabilize the endplate structures if there is excess cholinergic transmission resulting from long-term anticholinesterase medication. Source: Original.

out' found that this pathway works to counteract the intrinsic dispersal effect that neurotransmission has on AChR clusters and synaptic structure $[47,48]$. Long-term anticholinesterase therapy is known to affect neurotransmission and endplate fine structure $[49,50]$, and in some cases of AChR deficiency the early beneficial response to cholinesterase inhibitors becomes less effective with its longer term use [46"']. A plausible explanation for the beneficial effects of $\beta 2$-agonists is that they provide a compensatory mechanism to stabilize motor endplate structures through activation of second messenger signaling that partially compensates for the impairment in the AGRN-MuSK signaling pathway (Fig. 2). Similarly, $\beta 2$-agonists may help to counteract the disruptive effect on neuromuscular junction structure of long-term use of cholinesterase inhibitors in cases of AChR deficiency. This hypothesis would predict that the many subtypes of CMS that are responsive to anticholinesterase therapy and are on long-term anticholinesterase medication should be helped by the addition of $\beta 2$-agonists in their treatment regimen.

\section{CONCLUSION}

NGS is becoming an integral part of the diagnostic process for rare genetic disorders. Additional new CMS disease genes will be identified. Increasingly, disorders are now being identified in which impaired neuromuscular transmission is only one component in a more complex phenotypic spectrum. It is important to recognize the myasthenic component within these disorders as symptomatic treatment for the myasthenia is available. An expanding knowledge of the pathogenic mechanisms that underlie CMS helps in refining treatment combinations that can maximize the benefit to patients.

\section{Acknowledgements}

We gratefully acknowledge the UK National Specialised Commissioning Team for funding to the Diagnostic and Advisory service for CMS in Oxford.

\section{Financial support and sponsorship}

D.B. received support from $M R C$ programme grant $M R /$ M006824/1.

\section{Conflicts of interest}

There are no conflicts of interest.

\section{REFERENCES AND RECOMMENDED READING}

Papers of particular interest, published within the annual period of review, have been highlighted as

- of special interest

- of outstanding interest

1. Engel A, Shen X-M, Secen $D$, Sine S. Congenital myasthenic syndromes: pathogenesis, diagnosis, and treatment. Lancet Neurol 2015; 14:421-434.

2. Finlayson $S$, Beeson $D$, Palace J. Congenital myasthenic syndromes: an update. Pract Neurol 2013; 13:80-91.

3. Rodríguez Cruz PM, Palace J, Beeson D. Congenital myasthenic syndromes and the neuromuscular junction. Curr Opin Neurol 2014; 27:566-575.

4. Chaouch $A$, Beeson $D$, Hantaï $D$, Lochmüller $H$. 186th ENMC international workshop: congenital myasthenic syndromes 24-26 June 2011, Naarden, The Netherlands. Neuromuscul Disord 2012; 22:566-576.

5. Ohno K, Tsujino A, Brengman JM, et al. Choline acetyltransferase mutations cause myasthenic syndrome associated with episodic apnea in humans. Proc Natl Acad Sci U S A 2001; 98:2017-2022.

6. Arredondo J, Lara M, Gospe $\mathrm{S}$, et al. Choline acetyltransferase mutations causing congenital myasthenic syndromes: molecular findings and genotypephenotype correlations. Hum Mutat 2015; 36:881-893.

7. Shen XM, Selcen D, Brengman J, Engel AG. Mutant SNAP25B causes

myasthenia, cortical hyperexcitability, ataxia, and intellectual disability. Neurology $2014 ; 83: 2247-2255$.

Establishes a novel disorder in which of congenital myasthenia is part of a wider phenotypic spectrum arising from impaired neurotransmitter release due to defects of the SNARE complex.

8. Herrmann DN, Horvath R, Sowden JE, et al. Synaptotagmin 2 mutations

- cause an autosomal-dominant form of Lambert-Eaton myasthenic syndrome and nonprogressive motor neuropathy. Am J Hum Genet 2014; 95:332-339.

Establishes another presynaptic myasthenic syndrome with features similar to the autoimmune Lambert-Eaton myasthenic syndrome and is due to mutations in SYT2 which interacts with SNAP25B in the vesicular release of ACh from the presynaptic nerve terminal.

9. Whittaker RG, Herrmann DN, Bansagi B, et al. Electrophysiologic features of SYT2 mutations causing a treatable neuromuscular syndrome. Neurology 2015; 85:1964-1971.

10. Mohrmann $\mathrm{R}$, de Wit $\mathrm{H}$, Connell $\mathrm{E}$, et al. Synaptotagmin interaction with SNAP-25 governs vesicle docking, priming, and fusion triggering. J Neurosci 2013; 33:14417-14430.

11. Ohno K, Brengman J, Tsujino A, Engel AG. Human endplate acetylcholinesterase deficiency caused by mutations in the collagen-like tail subunit (COLQ) of the asymmetric enzyme. Proc Natl Acad Sci U S A 1998; 95:9654-9659.

12. Maselli RA, $\mathrm{Ng} J$ J, Anderson JA, et al. Mutations in LAMB2 causing a severe form of synaptic congenital myasthenic syndrome. J Med Genet 2009; 46:203-208. 
13. Logan CV, Cossins J, Rodríguez Cruz PM. Congenital myasthenic syndrome -1. type 19 is caused by mutations in COL13A1, encoding the atypical nonfibrillar collagen type XIII $\alpha 1$ chain. Am J Hum Genet 2015; 97:878-885. A new form of congenital myasthenic syndrome (CMS) due to mutations in the basal lamina protein collagen $13 \mathrm{~A} 1$, in which frameshift mutations disrupt development and maintenance of the neuromuscular junction. The work demonstrates that extracellular proteins not associated with the AGRN-MUSK signaling pathway play an important role in maintenance of synaptic structure.

14. Kvist AP, Latvanlehto $A$, Sund $M$, et al. Lack of cytosolic and transmembrane domains of type XIII collagen results in progressive myopathy. Am J Pathol 2001; 159:1581-1592.

15. Latvanlehto A, Fox MA, Sormunen R, et al. Muscle-derived collagen XIII regulates maturation of the skeletal neuromuscular junction. J Neurosci 2010; 30:12230-12241.

16. $\mathrm{Kim} \mathrm{N}$, Stiegler AL, Cameron TO, et al. Lrp4 is a receptor for Agrin and forms a complex with MuSK. Cell 2008; 135:334-342.

17. Zhang W, Coldefy A-S, Hubbard SR, Burden SJ. Agrin binds to the N-terminal region of Lrp4 protein and stimulates association between Lrp4 and the first immunoglobulin-like domain in muscle-specific kinase (MuSK). J Biol Chem $2011 ; 286: 40624-40630$.

18. Nicole S, Chaouch A, Torbergsen T. Agrin mutations lead to a congenital

myasthenic syndrome with distal muscle weakness and atrophy. Brain 2014; 137:2429-2443.

This report expands the phenotypic spectrum that can result from mutations in AGRN and shows that AGRN mutations can give rise to a distal myopathy.

19. Selcen $D$, Ohkawara $B$, Shen $X M$, et al. Impaired synaptic development,

- maintenance, and neuromuscular transmission in LRP4-related myasthenia. JAMA Neurol 2015; 72:889-896.

A second case of a CMS due to mutations in LRP4, confirming the crucial role of LRP4 in the development and maintenance of the neuromuscular junction and highlighting a potential hotspot for CMS mutations within LRP4 located at the edge of its third beta-propeller domain.

20. Ohkawara B, Cabrera-Serrano M, Nakata T, et al. LRP4 third $\beta$-propeller domain mutations cause novel congenital myasthenia by compromising agrinmediated MuSK signaling in a position-specific manner. Hum Mol Genet 2014; 23:1856-1868.

21. Li $Y$, Pawlik B, Elcioglu $N$, et al. LRP4 mutations alter $\mathrm{Wnt} /$ beta-catenin signaling and cause limb and kidney malformations in Cenani-Lenz syndrome. Am J Hum Genet 2010; 86:696-706.

22. Leupin $\mathrm{O}$, Piters $\mathrm{E}$, Halleux $\mathrm{C}$, et al. Bone overgrowth-associated mutations in the LRP4 gene impair sclerostin facilitator function. J Biol Chem 2011; 286:19489-19500.

23. Jaeken J, Matthijs G. Congenital disorders of glycosylation. Annu Rev Genomics Hum Genet 2001; 2:129-151.

24. Wu X, Rush JS, Karaoglu D, et al. Deficiency of UDP-GlcNAc:Dolichol phosphate $N$-acetylglucosamine-1 phosphate transferase (DPAGT1) causes a novel congenital disorder of Glycosylation Type lj. Hum Mutat 2003; 22:144-150.

25. Thiel C, Schwarz M, Peng J, et al. A new type of congenital disorders of glycosylation (CDG-li) provides new insights into the early steps of dolichollinked oligosaccharide biosynthesis. J Biol Chem 2003; 278:22498-22505.

26. Belaya $K$, Finlayson $S$, Slater $C R$, et al. Mutations in DPAGT1 cause a limbgirdle congenital myasthenic syndrome with tubular aggregates. Am J Hum Genet 2012; 91:193-201.

27. Senderek J, Müller JS, Dusl M, et al. Hexosamine biosynthetic pathway mutations cause neuromuscular transmission defect. Am J Hum Genet 2011; 88:162-172.

28. Cossins J, Belaya K, Hicks D, et al. Congenital myasthenic syndromes due to mutations in ALG2 and ALG14. Brain 2013; 136:944-956.

29. Chen $Q$, Müller JS, Pang PC, et al. Global N-linked glycosylation is not significantly impaired in myoblasts in congenital myasthenic syndromes caused by defective glutamine-fructose-6-phosphate transaminase 1 (GFPT1). Biomolecules 2015; 5:2758-2781.

30. Zoltowska K, Webster R, Finlayson S, et al. Mutations in GFPT1 that underlie limb-girdle congenital myasthenic syndrome result in reduced cell-surface expression of muscle AChR. Hum Mol Genet 2013; 22:2905-2913.

31. Dusl M, Senderek J, Müller JS, et al. A $3^{\prime}$-UTR mutation creates a microRNA

- target site in the GFPT1 gene of patients with congenital myasthenic syndrome. Hum Mol Genet 2015; 24:3418-3426.

This study demonstrates a novel molecular mechanism through which sequence variants might cause a myasthenic syndrome - namely through creating or deleting micoRNA binding sites. It will be important to bear this in mind in cases where the search for causative mutations has proved elusive.
32. Ning $B$, Elbein $A D$. Cloning, expression and characterization of the pig liver GDP-mannose pyrophosphorylase. Evidence that GDP-mannose and GDPGlu phosphorylases are different proteins. Eur J Biochem/FEBS 2000; 267:6866-6874

33. Carss KJ, Stevens E, Foley AR, et al. Mutations in GDP-mannose pyrophosphorylase B cause congenital and limb-girdle muscular dystrophies associated with hypoglycosylation of $\alpha$-dystroglycan. Am J Hum Genet 2013; 93:29-41.

34. Cabrera-Serrano $M$, Ghaoui $R$, Ravenscroft $G$, et al. Expanding the phenotype of GMPPB mutations. Brain 2015; 138:836-844.

35. Belaya K, Rodríguez Cruz PM, Liu WW, et al. Mutations in GMPPB cause

-1. congenital myasthenic syndrome and bridge myasthenic disorders with dystroglycanopathies. Brain 2015; 138:2493-2504.

Establishes mutations in GMPPB as a cause of CMS. This is an enzyme that can affect the early steps of the $\mathrm{N}$-glycosylation and $\mathrm{O}$-glycosylation pathways and causes phenotypes in which myasthenia and a dystroglycanopathy can overlap.

36. Rodriguez Cruz PM, Belaya $\mathrm{K}$, Basiri $\mathrm{K}$, et al. Clinical features of the myasthenic syndrome arising from mutations in GMPPB. J Neurol Neurosurg Psychiatry 2016; 87:802-809.

37. Arnold W, Feldman D, Ramirez $S$, et al. Defective fast inactivation recovery

- of $\mathrm{Na}_{\mathrm{v}} 1.4$ in congenital myasthenic syndrome. Ann Neurol 2015; 77:840850.

Identification of a rare form of CMS and a detailed study into the mechanisms through which mutations in SCNA4 that is present within the postsynaptic fold at the endplate can result in myasthenic weakness.

38. Habbout $\mathrm{K}$, Poulin $\mathrm{H}$, Rivier $\mathrm{F}$, et al. A recessive $\mathrm{Na}_{\mathrm{v}} 1.4$ mutation underlies

- congenital myasthenic syndrome with periodic paralysis. Neurology 2016; 86:161-169.

Another SCN4A mutation, though in this case clinical features of myasthenia and periodic paralysis overlap.

39. Todd E, Yau K, Ong R, et al. next generation sequencing in a large cohort of patients presenting with neuromuscular disease before birth. Orphanet J Rare Dis $2015 ; 10: 148$.

40. Garg N, Yiannikas $C$, Hardy TA. Late presentations of congenital myasthenic syndromes - how many do we miss? Muscle Nerve 2016. [Epub ahead of print]

41. Lashley D, Palace J, Jayawant $\mathrm{S}$, et al. Ephedrine treatment in congenital myasthenic syndrome due to mutations in DOK7. Neurology 2010; 74:1517-1523.

42. Lorenzoni PJ, Scola RH, Kay CSK, et al. Salbutamol therapy in congenital myasthenic syndrome due to DOK7 mutation. J Neurol Sci 2013; 331:155157.

43. Burke G, Hiscock A, Klein A, et al. Salbutamol benefits children with congenital myasthenic syndrome due to DOK7 mutations. Neuromuscul Disord 2013; 23:170-175.

44. Witting $N$, Vissing J. Pharmacologic treatment of downstream of tyrosine kinase 7 congenital myasthenic syndrome. JAMA Neurol 2014; 71:350354

45. Gallenmuller C, Muller-Felber W, Dusl M, et al. Salbutamol responsive limb-girdle congenital myasthenic syndrome due to novel missense mutation and heteroallelic deletion in MUSK. Neuromuscul Disord 2014; $24: 31-35$.

46. Rodríguez Cruz PM, Palace J, Ramjattan $\mathrm{H}$, et al. Salbutamol and ephedrine in

a. the treatment of severe AChR deficiency syndromes. Neurology 2015; 85:1043-1047.

A clinical study that shows the dramatic beneficial effects of adding salbutamol to patients with severe disability due to AChR deficiency syndrome even though the standard treatment with pyridostigmine and 3,4-DAP had been optimized. The authors suggest $\beta 2$-agonists should be beneficial as an add-on therapy to any patients on long-term anticholinesterase medication.

47. Misgeld T, Kummer TT, Lichtman JW, Sanes JR. Agrin promotes synaptic differentiation by counteracting an inhibitory effect of neurotransmitter. Proc Natl Acad Sci U S A 2005; 102:11088-11093.

48. Kummer TT, Misgeld T, Sanes JR. Assembly of the postsynaptic membrane at the neuromuscular junction: paradigm lost. Curr Opin Neurobiol 2006; 16:74-82.

49. Engel AG, Lambert EH, Santa T. Study of long-term anticholinesterase therapy: effects on neuromuscular transmission and on motor endplate fine structure. Neurology 1973; 23:1273-1281.

50. Gillies JD, Allen J. Effects of neostigmine and pyridostigmine at the neuromuscular junction. Clin Exp Neurol 1977; 14:271-279. 